\title{
Immature Neutrophil Count
}

National Cancer Institute

\section{Source}

National Cancer Institute. Immature Neutrophil Count. NCI Thesaurus. Code C96678.

The determination of the amount of immature neutrophils present in a sample. 